\title{
Design and Fabrication of CLYC-Based Rotational Modulation Collimator (RMC) System for Gamma-Ray/Neutron Dual-Particle Imager
}

\author{
Hyun Suk Kim', Jooyub Lee², Sanghun $\mathrm{Choi}^{3}$, Young-bong Bang ${ }^{4}$, Sung-Joon $\mathrm{Ye}^{5}$, Geehyun $\mathrm{Kim}^{6}$ \\ ${ }^{1}$ Division of Radiation Regulation, Korea Institute of Nuclear Safety (KINS), Daejeon, Korea; ${ }^{2}$ Department of Nuclear Engineering, Sejong University, Seoul, \\ Korea; ${ }^{3}$ Division of Technology Planning Management, Korea Research Institute for defense Technology planning and advancement (KRIT), Jinju, Korea; \\ ${ }^{4}$ Transdisciplinary Department of Medicine and Advanced Technology, Seoul National University Hospital, Seoul, Korea; ${ }^{5}$ Department of Applied Bioengineering, \\ Graduate School of Convergence Science and Technology, Seoul National University, Seoul, Korea; ${ }^{6}$ Department of Nuclear Engineering, Seoul National \\ University, Seoul, Korea
}

\section{Original Research}

Received June 14, 2021

Revision August 11, 2021

Accepted September 2, 2021

Corresponding author: Geehyun Kim

Department of Nuclear Engineering, Seoul National University, 1 Gwanak-ro,

Gwanak-gu, Seoul 08826, Korea

E-mail: gk.rs@snu.ac.kr

(D) https://orcid.org/0000-0003-4302-2659

\begin{abstract}
This is an open-access article distributed under the terms of the Creative Commons Attribution License (http://creativecommons.org/licenses/by-nc/4.0), which permits unrestricted use, distribution, and reproduction in any medium, provided the original work is properly cited.
\end{abstract}

Copyright $\odot 2021$ The Korean Association for Radiation Protection

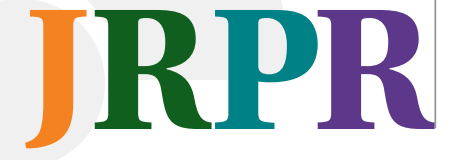

Background: This work aims to develop a new imaging system based on a pulse shape discrimination-capable $\mathrm{Cs}_{2} \mathrm{LiYCl}_{6}$ : $\mathrm{Ce}$ (CLYC) scintillation detector combined with the rotational modulation collimator (RMC) technique for dual-particle imaging.

Materials and Methods: In this study, a CLYC-based RMC system was designed based on Monte Carlo simulations, and a prototype was fabricated. Therein, a rotation control system was developed to rotate the RMC unit precisely, and a graphical user interface-based software was also developed to operate the data acquisition with RMC rotation. The RMC system was developed to allow combining various types of collimator masks and detectors interchangeably, making the imaging system more versatile for various applications and conditions.

Results and Discussion: Operational performance of the fabricated system was studied by checking the accuracy and precision of the collimator rotation and obtaining modulation patterns from a gamma-ray source repeatedly.

Conclusion: The prototype RMC system showed reliability in its mechanical properties and reproducibility in the acquisition of modulation patterns, and it will be further investigated for its dual-particle imaging capability with various complex radioactive source conditions.

Keywords: Radiation Imaging, Rotational Modulation Collimator (RMC), Dual-Particle Imager, CLYC Scintillation Detector, Radioactivity Monitoring

\section{Introduction}

In the fields of homeland security and nuclear safety, much effort has been expended on the radiation imaging technique. Most of imagers use spatially or temporally encoded information of incident particles to estimate the location of radioactivity [1, 2]. A coded-aperture imager is a typical example of radiation imager based on mechanical collimation using a mask consisting of opaque and transparent pixels systematically arranged [1]. Analyzing the shadow pattern cast by the coded-aperture on a positionsensitive detector estimates the original image of the radioactivity distribution. Another common type of gamma-ray imager is the Compton camera, and it is based on Compton scattering events of incoming radiation [2]. The scattering of primary particles oc- 
curs in the first detection plane recording the position and energy of the recoil electron, and it continues to proceed toward the second detection plane where it is often absorbed, recording the position and energy of the scattered gamma. However, both techniques require position-sensitive radiation detectors which either exist as an array of several detectors or made as a pixelated or a double-strip type device, of which the complexity and the cost of the system fabrication are often significant.

The rotational modulation collimator (RMC)-based imaging technique is one of the indirect imaging methods, converting non-image data such as modulation patterns to radiation images using a source-distribution-mapping algorithm. In terms of the simplicity and cost of the system, the RMC technique could be an attractive candidate. This technique, which is based on temporal modulation of incident radiation signals, does not require position-sensitive radiation detectors and thus, excludes the necessity of complex electronics and requires less fabrication cost [3-7]. This imager estimates the radioactive source distribution from the pattern morphology determined by the system configuration of the RMC imager and by the individual source location. As the RMC rotates, the intensity of the incoming flux of particles to the detector changes over time, depending on the open area created by collimator masks, and the detector outputs are processed to generate modulation patterns that can be used to reconstruct images of radioactivity distribution.

The RMC technique has been around since the 1960s in the astronomy field, and it has expanded into the field of medical imaging and homeland security [3-5]. However, previous RMC studies are limited to only single-particle imaging system based on the gamma-ray measurement. As an attempt to develop a gamma-ray/neutron dual-particle imager for compensating the drawbacks of the single-particle imager, we employed RMC coupled to a pulse shape discrimination (PSD)-capable CLYC $\left(\mathrm{Cs}_{2} \mathrm{LiYCl}_{6}: \mathrm{Ce}\right)$ detector [6, 7]. In our system, the CLYC detector consists of a $2 \times 2$ inch cylindrical CLYC crystal (95\% enriched ${ }^{6} \mathrm{Li}$ ), coupled to a R6233-100 photomultiplier tube (Hamamatsu Photonics, Japan) and signal processing electronics for charge comparison method (Fig. 1) [8].

The RMC imager developed in the current work bases on the CLYC-6 (95\% enriched ${ }^{6} \mathrm{Li}$ ) detector which is particularly sensitive to slow neutrons in addition to the gamma-ray spectroscopy capability with a reasonable energy resolution, and the dual-particle imaging performance of the developed sys-

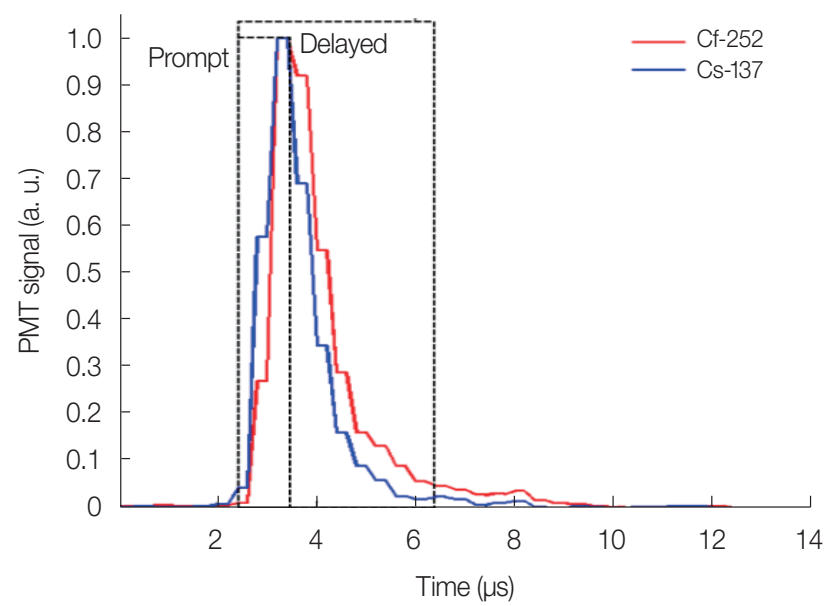

Fig. 1. Illustration of pulse shape discrimination technique (charge comparison method): comparison of detector pulses integrated over two different intervals as prompt and delayed signal. PMT, photomultiplier tube.

tem is anticipated to be mostly aimed at gamma-ray and slow neutron imaging. However, one of the important design considerations in the current prototype development is the interchangeability of the collimator masks and the main detector, which can improve the versatility of the RMC prototype system. In this regard, the RMC system can be easily transformed into a gamma-ray/fast neutron dual-particle imaging system by adopting an organic scintillator or CLYC-7 (99\% enriched ${ }^{7} \mathrm{Li}$ ) detector as the main detector of the imager combined with the PSD technique [9]. In the current work, we investigated design parameters for the RMC system and fabricated a prototype system for the dual-particle imaging. The developed system was examined for its mechanical properties such as rotation accuracy and was also tested with a gamma-ray source to confirm pattern obtainability and reproducibility.

\section{Materials and Methods}

\section{Design of the RMC Structure and Mask}

A schematic of the RMC system prototype is shown in Fig. 2A. The RMC system consists of an aluminum support tube, a driver tube with two ball bearings, two collimator masks, a timing pulley/belt assembly, a stepper motor and a CLYC detector. On the driver tube, there are four pins installed respectively at the front and rear ends. One can attach and replace collimator masks with the help of these pins, which make the collimator masks interchangeable for the RMC system. A stepper motor was mounted on the top of the driver 

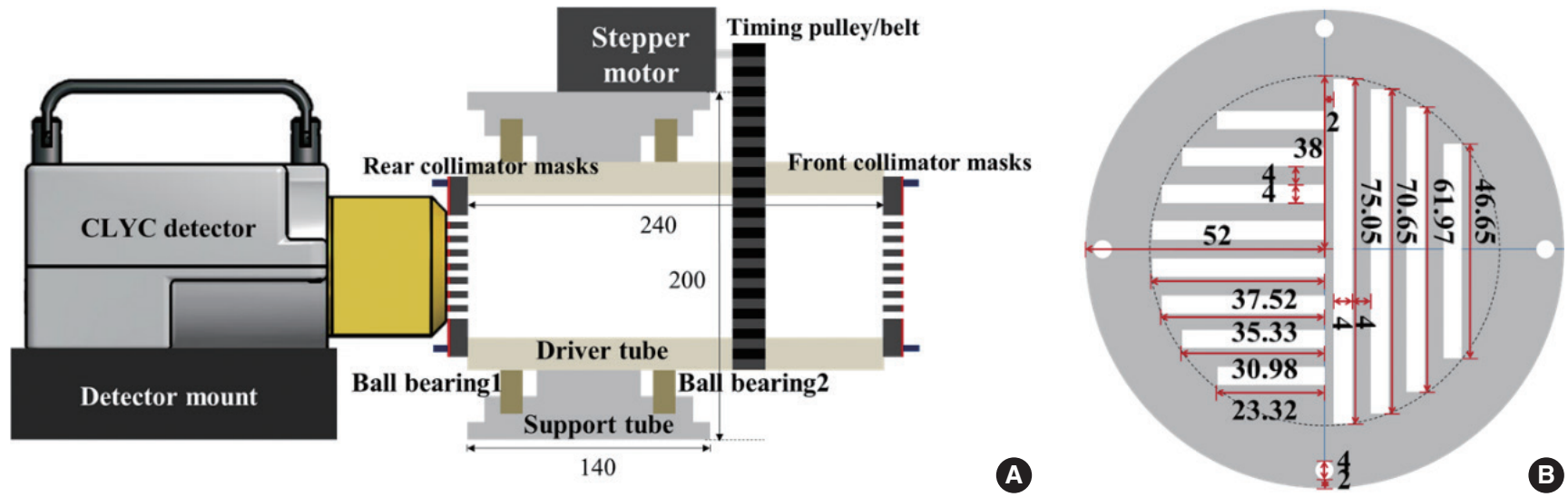

Fig. 2. (A) The design of $\mathrm{Cs}_{2} \mathrm{LiYCl}_{6}: \mathrm{Ce}$ (CLYC)-based rotational modulation collimator (RMC) structure and (B) a collimator mask (unit: mm).
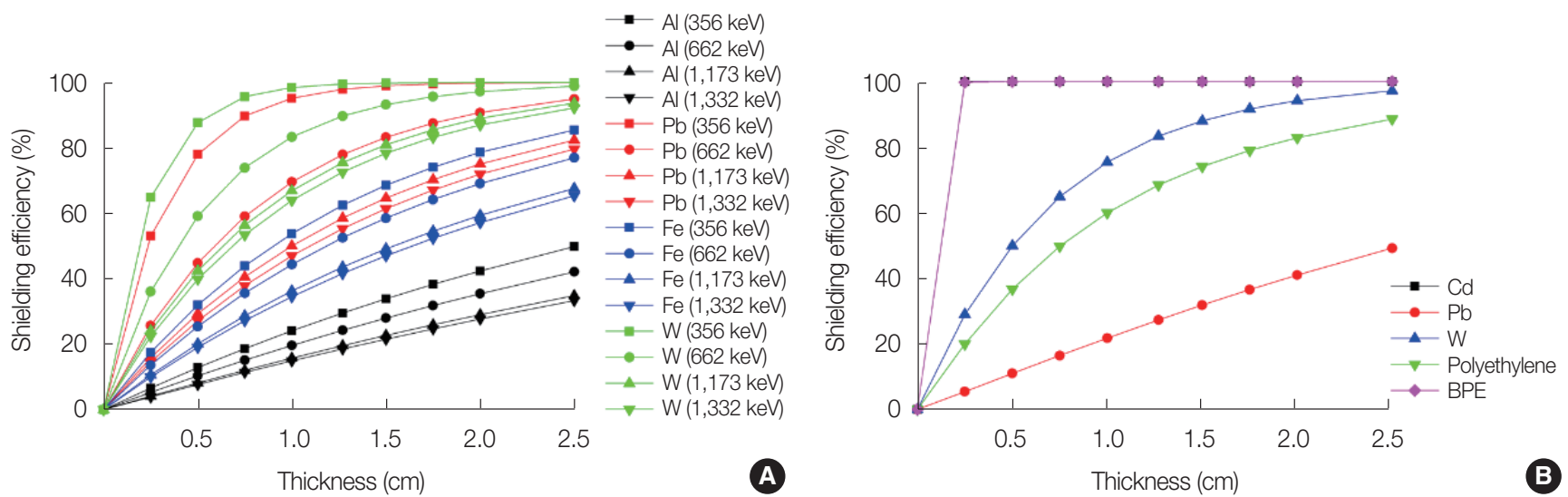

Fig. 3. Shielding efficiencies versus mask thickness and materials: (A) gammas and (B) thermal neutrons. BPE, borated polyethylene.

tube to rotate the collimator masks, and timing pulleys and belt were used to transmit the power from the stepper motor. The timing pulleys were designed to have the conversion factor of 4.5 to 1 , from the motor pulley to the driver tube pulley. That is, when the motor pulley rotates $4.5^{\circ}$, the driver tube rotates by $1^{\circ}$ along with the two collimator masks. In addition, a block mount was designed to adjust the height of the detector, so that the central axis of the detector crystal could be aligned to the center of the both collimator masks.

Collimator masks are the most important part of the RMC system as they essentially determine entire characteristics of the modulation pattern as well as the field-of-view (FOV) of the imager and the detection time required for imaging. First, for better quality and efficient imaging, it is desirable that the opacity of the collimator masks to incident radiations is high as possible. In order to determine a proper material candidate and thickness of the collimator masks for the dual-particle imager, Monte Carlo simulations were performed to calculate the shielding performance of the collimator using the Monte Carlo N-Particle Version 6.1 (MCNP6.1) code [10]. In the simulation, the detector was exposed to a point source of gamma rays and $0.025 \mathrm{eV}$ thermal neutrons that was located $25 \mathrm{~cm}$ away from the center of the cylindrical detector face. The gamma-ray response of the detector was calculated using the pulse height (F8) tally, and the neutron response in CLYC was counted by the cell flux tally with reaction multipliers (F4/FM) in MCNP6.1. The simulation was conducted for $1 \times 10^{8}$ source particle histories, and calculated results are shown in Fig. 3. Based on the shielding efficiencies, we designed a laminated structure of the collimator mask which is a combination of $1 \mathrm{~cm}$-thick lead $(\mathrm{Pb})$ and $0.2 \mathrm{~cm}$-thick borated polyethylene (BPE). In the MCNP6.1 simulation, the shielding efficiency of a $1 \mathrm{~cm}$-thick Pb mask was $95.20 \%$ for $356 \mathrm{keV}$ gamma rays, and the shielding efficiency for thermal neutron was $100 \%$ when $0.2 \mathrm{~cm}$-thick BPE was used.

As for the design of the collimator mask pattern, a common one is the bilaterally symmetric slits engraved on a circularshape mask. However, the design poses an intrinsic artifact 
problem in the image reconstruction stage due to the $180^{\circ}$ periodicity of the modulation pattern, inherent in the bilateral symmetry of slits [6]. The ambiguity in the source location estimation because of this artifact can be removed by employing an asymmetric slit pattern [11]. In the previous study, we investigated various designs of collimator mask patterns with bilateral asymmetry and optimized the asymmetric mask design, by adjusting slit/slat width and pitch. Based on our previous study and further MCNP6.1 simulations, we fabricated the collimator mask as shown in Fig. 2B [11].

\section{Fabrication of the RMC Structure and Mask}

Each component of the RMC structure was designed considering the accuracy of mechanical control, durability, and convenient operation. Components comprising the RMC system are shown in Fig. 4. Each part was manufactured individually and then assembled altogether. For the collimator mask, we designed the mask made of a laminated structure for the dual-particle collimating purpose as mentioned in the previous section. We used a $99.96 \%$ ultra-pure lead plate (Semyoung Metal, Gimpo, Korea) and a high-quality BPE plate (Dynex, Incheon, Korea) as base materials. The BPE plate has a higher hydrogen content $(>115 \mathrm{~g} / \mathrm{L}$ ) than water does, and the boron content of the plate is over $56 \mathrm{~g} / \mathrm{L}$. The slits of mask were cut with a lathe and a two-dimensional (2D) computer numerical control machine tool.
The driver/support tube was made of Aluminum Alloy 3003 (AA3003) because of its high strength and good resistance to atmospheric corrosion. Considering the driver tube size, we chose a 68-24-ZZ/C3 ball bearing (NJL Japan, Japan) which has $120 \mathrm{~mm}$ internal diameter and $150 \mathrm{~mm}$ external diameter for the rotation of the driver tube, and the bearings were fixed using a NR-145 snap ring (NTN, USA). The bearing housings were installed in the support tube to prevent the dislocation of the bearings.

We implemented a 22-tooth timing pulley (8 $\mathrm{mm}$ in internal diameter and $33.87 \mathrm{~mm}$ in external diameter) and a 99-tooth timing pulley (120 $\mathrm{mm}$ in internal diameter and in $156.41 \mathrm{~mm}$ in external diameter) to transfer the work load from the motor to the driver tube rotation, and the conversion factor from the motor pulley to the driver tube pulley is 4.5 to 1 . Considering the distance between the center of the driver tube and the stepper motor (i.e., $134.5 \mathrm{~mm}$ ), the belt was fabricated with the length of $600 \mathrm{~mm}$ and the width of 10 $\mathrm{mm}$. In order to prevent tension changes on the belt due to the experimental condition, we designed and fabricated a stepper motor mount which can adjust $\pm 2 \mathrm{~mm}$ of the distance between center of the driver tube and stepper motor.

Overall weight of the equipment is approximately $15 \mathrm{~kg}$ except for the detector, which would be appropriate to be developed as a mounted device on unmanned ground or marine vehicles. The overall weight of the system can be reduced

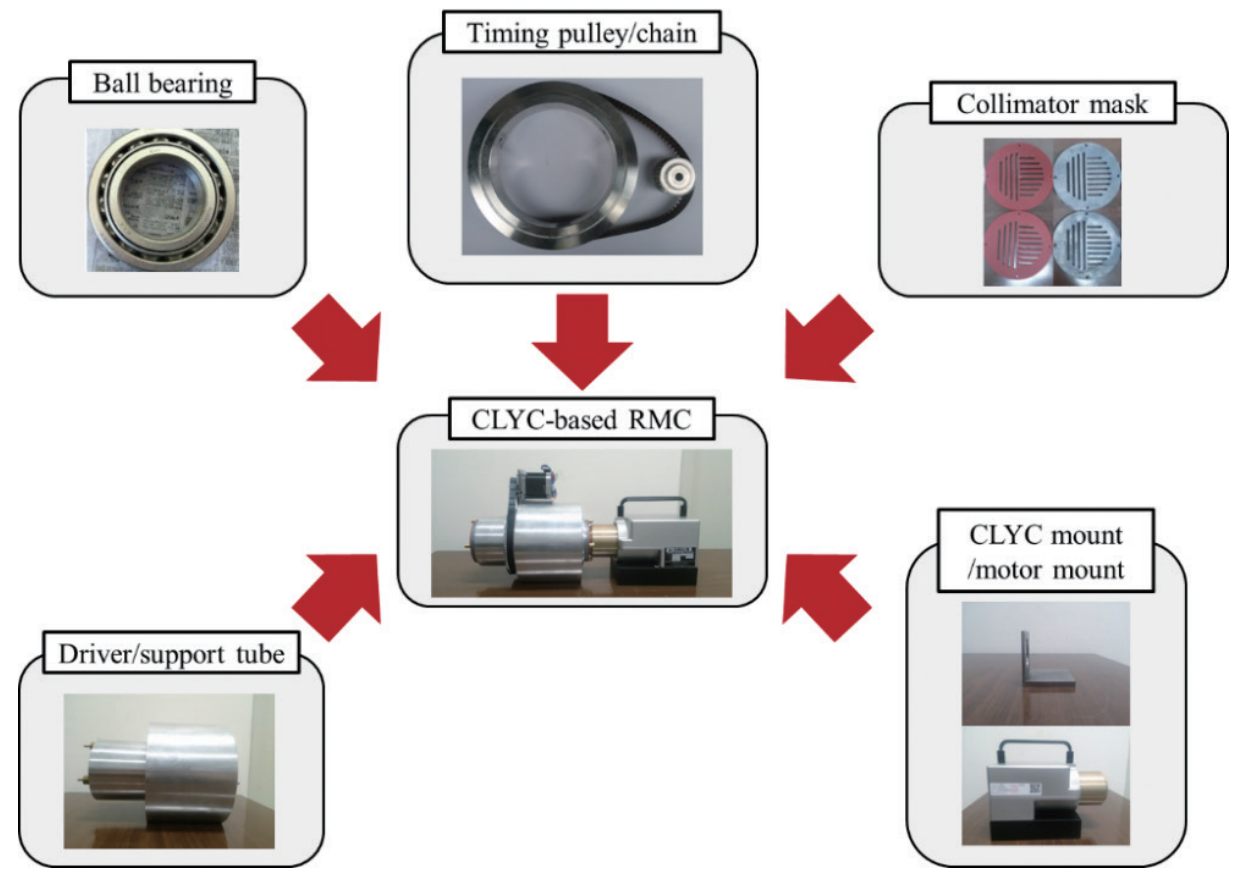

Fig. 4. Components of the Cs 2 LiYCla:Ce (CLYC)-based rotational modulation collimator (RMC) system. 
by replacing the driver tube made of solid aluminum with hollow body, and, depending on the selection of the main detector module, it can also be developed as a hand-held device without much complexity, as it does not require a position-sensitive detection system for the RMC imager. On the contrary, the system can be modified as an omni-directional imager, which features an extended FOV as large as approximately $2 \pi$, by implementing side slits on the driver tube $[12,13]$.

\section{Rotation Control System}

To control the rotation of the collimator masks, we used a QSH 6018-86-28-310 stepper motor (Trinamic Motion Control, Hamburg, Germany) controlled by an Arduino Due board connected to a DRV8880 motor driver (Pololu Robotics and Electronics, Las Vegas, NV, USA) which is capable of micro stepping with a minimum limit of $0.025^{\circ}$ per step. An RMC control console shown in Fig. 5 was also developed to protect the circuit from the heat and the mechanical shock, and to ease the control of the collimator rotation. The control console frame was designed with a three-dimensional (3D) computer-aided design (CAD) software and was 3D-printed (Ultimaker2; Brule, UTRECHT, Netherlands) with plastic. One can toggle options to control the collimator rotation either automatically or manually, as well as to change the direction of the rotation. The manual control mode is to be used for the collimator mask alignment procedure, and the automatic control mode is mostly to be used for the mea-

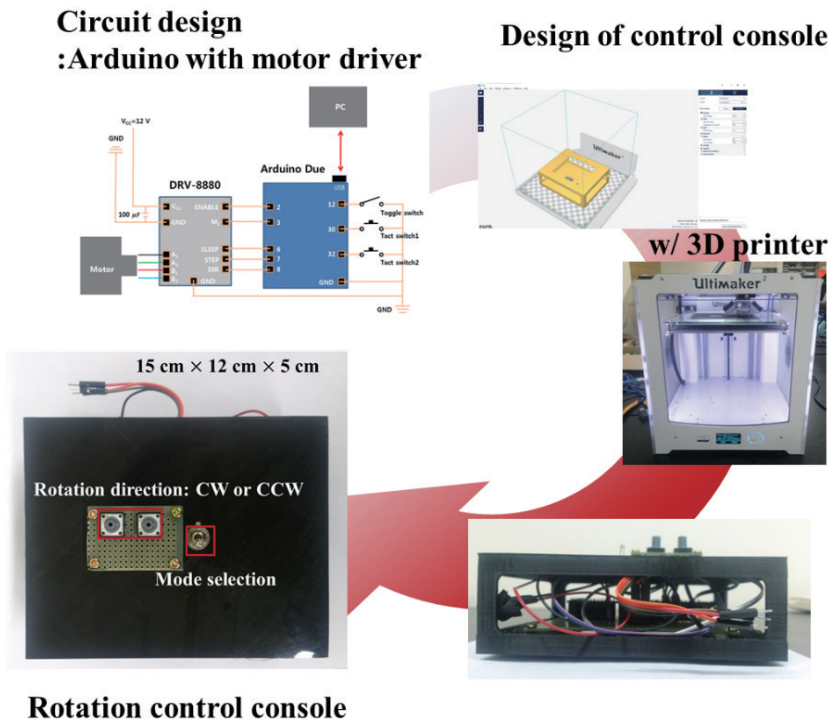

Fig. 5. Composition and the layout of the rotation control console. CW, clockwise; CCW, counterclockwise. surement experiments. In the automatic mode, one can repeat the rotation of collimator masks with a certain time interval and the rotation angle. The direction of the rotation (i.e., clockwise or counterclockwise) can be alternated with a tact switch.

A software to control the rotational system was developed using Arduino Sketch, a program written in the integrated development environment (IDE). We additionally developed a graphical user interface (GUI)-based software based on LabVIEW (National Instruments, Austin, TX, USA) for the serial communication between the Arduino and the computer. It provides an easy-to-use GUI environment to monitor the status of the rotation, in which it will show the rotational information such as the operation mode, rotation direction, rotation interval, and the current time and position updated in real-time, and it also provides a functionality of saving the information in a text format. A screenshot of the developed rotation control software is shown in Fig. 6.

\section{Results and Discussion}

\section{Rotation Accuracy}

The rotation accuracy is practically important to obtain accurate and reproducible modulation patterns, which are di-

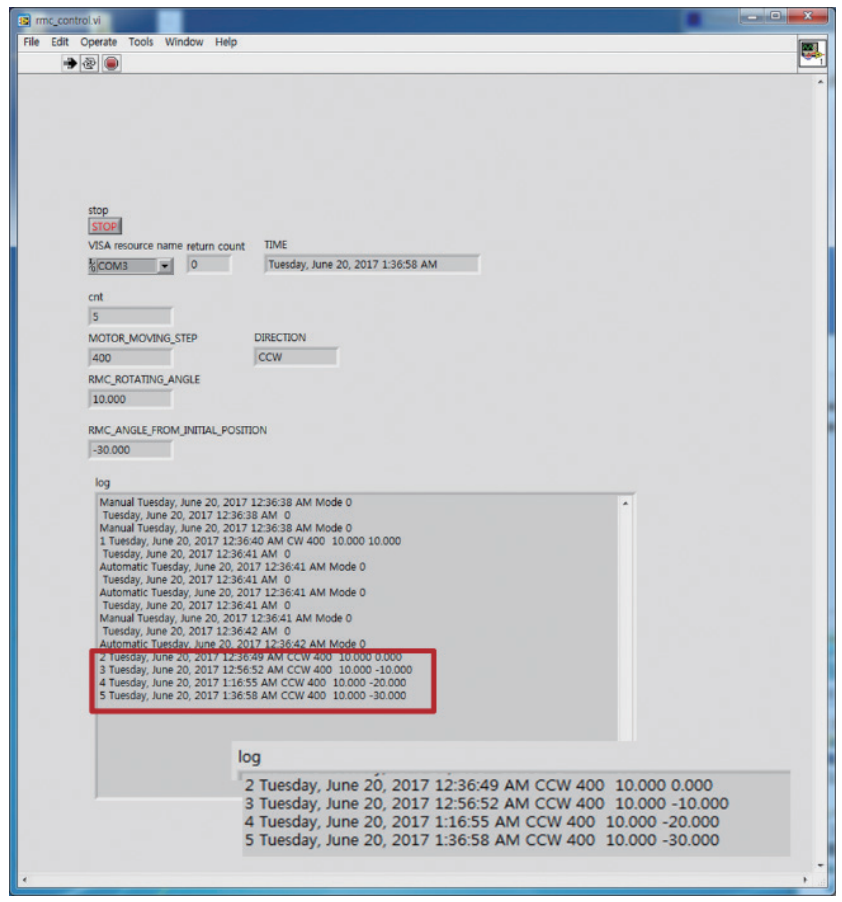

Fig. 6. An execution window of the rotational modulation collimator rotation control software. 

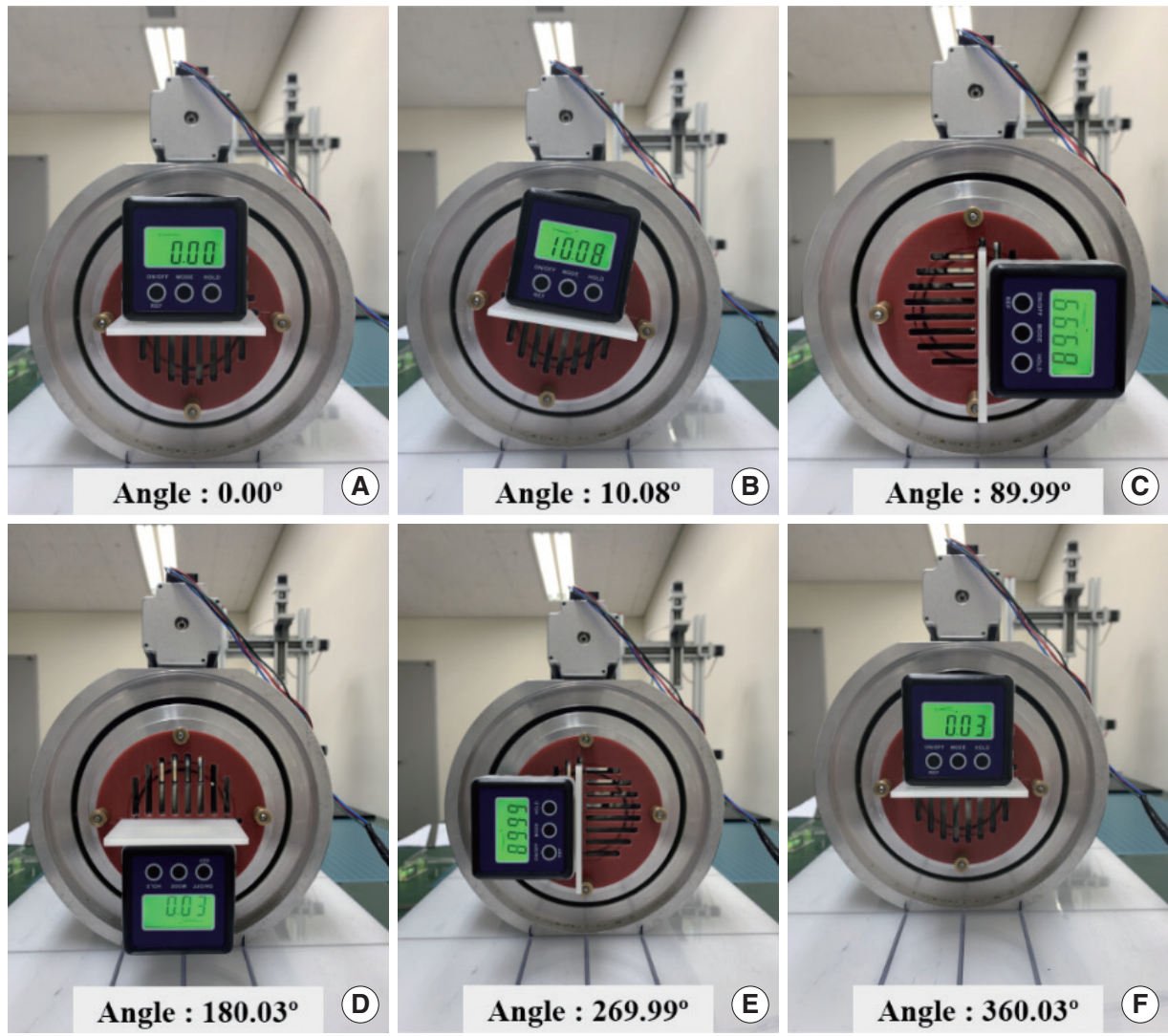

Fig. 7. Rotation accuracy test for the nominal rotation angles of: (A) $0^{\circ}$, (B) $10^{\circ}$, (C) $90^{\circ}$, (D) $180^{\circ}$, (E) $270^{\circ}$, and (F) $360^{\circ}$.

rectly related to the quality of reconstructed images. The rotation angle of the collimators was physically calibrated and investigated using a digital protractor and flat bar $(350 \times 70 \times$ $4 \mathrm{~mm}^{3}$ ), which tightly fits the mask slit as shown in Fig. 7. The flat bar was inserted into a slit at the front mask, fed through the driver tube, and inserted into the corresponding slit on the rear mask. Rotating collimators with a $10^{\circ}$ interval, we repeatedly measured the angles of the flat bar using the digital protractor. The measurement was repeated for five times with a set of rotating collimator masks by a $360^{\circ}$ full rotation. At each rotation angle, measured angles indicated on the protractor were compared with the nominal angle displayed on the GUI-based software, and the relative error from the nominal value was calculated. Throughout the five rounds of full rotation with $10^{\circ}$ interval, each measurement of the angle matched nominal angle values displayed on the computer screen, within the error of $0.1 \%$, as shown in Fig. 7 .

\section{Reproducibility of the Pattern}

For the measurement experiments, a $10.64 \mu \mathrm{Ci}{ }^{133} \mathrm{Ba}$ source (Eckert \& Ziegler, Berlin, Germany) calibrated on April 1, 2016 was placed at $(5 \mathrm{~cm}, 0 \mathrm{~cm}, 100 \mathrm{~cm})$. The $(0 \mathrm{~cm}, 0 \mathrm{~cm}, 0 \mathrm{~cm})$ position was set to the rotation axis of the front mask face. At each rotation angle, we collected gamma-ray energy spectra obtained by the CLYC detector for 20 minute-dwell time, and calculated the net peak area of $356 \mathrm{keV}$ photopeak from each spectrum. The modulation pattern is constructed from the net peak areas calculated at each rotation angle. The measurement was performed for several sets, each set consisting of 36 gamma-ray spectra measured with $10^{\circ}$ rotation interval. Fig. 8 shows three modulation patterns obtained from the ${ }^{133} \mathrm{Ba}$ source measurements compared to the pattern calculated by the MCNP6.1 simulation. Even though one could observe slight inconsistency due to the statistical error of gamma-ray counting, measured modulation patterns showed reasonable reproducibility in the overall shape of the modulation pattern and are well matched to the simulated pattern obtained by MCNP6.1. Measurement experiments gave promising results on the CLYC-based RMC system as a radiation imager, considering that the reproducibility of the modulation patterns and the agreement with the simulation result can favor the quality of the reconstructed images. 


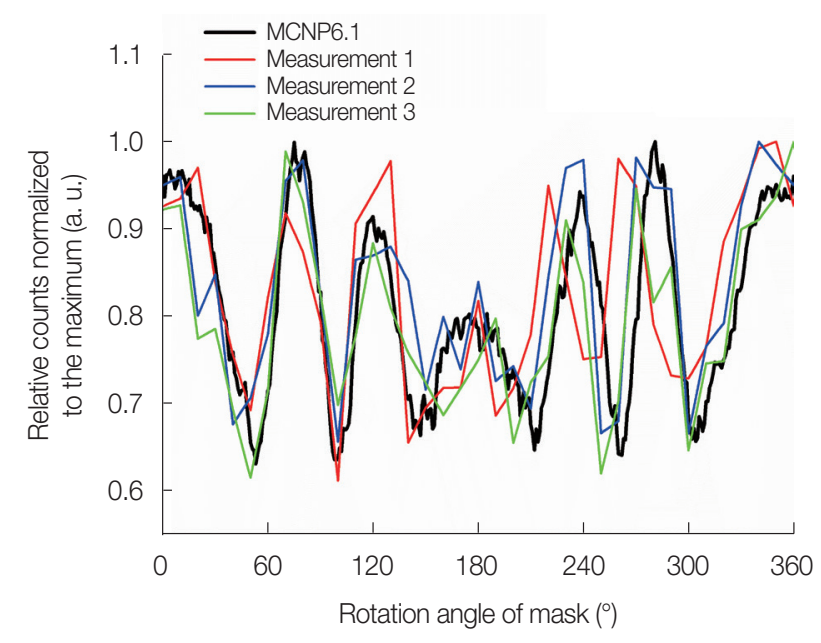

Fig. 8. Modulation patterns obtained by ${ }^{133} \mathrm{Ba}$ source measurement experiments and Monte Carlo N-Particle Version 6.1 (MCNP6.1) simulation.

\section{Discussion}

In this paper, we mainly focused on the design and the fabrication of the CLYC-based RMC system for the dual-particle imaging purpose, and on the demonstration of feasibility to be utilized for a radiation imager. There could be various types of applications for the dual-particle imager, and some applications may require locating a trace amount of radioactive material less than $1 \mathrm{~g}$ or less. A practical issue encountered in the current study was the long detection time for constructing the modulation patterns because of the low activity of the source used in the measurement experiment. The total acquisition time of the modulation pattern took approximately 12 hours to obtain a modulation pattern. However, considering that finding the distribution of an approximately $10 \mu \mathrm{Ci}$ source is not a practical scope of interest for a radiation imager, we can expect the dwell time would dramatically decrease in a realistic situation involving a high activity source. For example, the specific activity of ${ }^{235} \mathrm{U}$ is 2.2 $\mu \mathrm{Ci} / \mathrm{g}$ and those of ${ }^{238} \mathrm{Pu},{ }^{239} \mathrm{Pu},{ }^{240} \mathrm{Pu}$, and ${ }^{241} \mathrm{Pu}$ are $17.3 \mathrm{Ci} / \mathrm{g}$, $0.063 \mathrm{Ci} / \mathrm{g}, 0.23 \mathrm{Ci} / \mathrm{g}$, and $104 \mathrm{Ci} / \mathrm{g}$, respectively. If the radioactivities of significant quantities of special nuclear materials are calculated, they are expected to be at least $50 \mathrm{mCi}$ or much larger. Therefore, even though the CLYC-based RMC system developed in this work might not be optimal for tracing a trace-amount of the nuclear material which is carried by private parties, we envision the application to the first-line detection and surveillance of nuclear activity by monitoring the illegal flow of nuclear materials, in which the total detection time is expected to decrease to an order of a few minutes.
In the following study, we plan to test our RMC system in more complex radiation environments including multiple gamma-ray sources as well as neutron sources mixed with gamma-ray sources. Based on the measured modulation patterns, 2D radiation images will be reconstructed and the imaging performance of the CLYC-based RMC system will be evaluated. Maximum-likelihood expectation-maximization (MLEM) algorithm-based image reconstruction methods have been extensively studied and utilized in the field of radiation imaging because of their inherent excellence in low-count high-noise problems [7, 11-17]. We have been developing techniques which can further improve the efficiency and performance of the MLEM-based image reconstruction algorithm, by stabilizing inherent statistical noise contributions from the radiation counting [18-20]. Newly developed reconstruction algorithms require less iteration to converge whilst achieving even better imaging performance in terms of the signal-to-noise ratio and the structural similarity index. In this regard, one can expect to reduce the overall detection and calculation time even more to obtain the radiation image.

\section{Conclusion}

In this study, a CLYC-based RMC system capable of dualparticle imaging was designed and fabricated. The system consists of aluminum support/driver tubes with two ball bearings, two collimator masks, a timing pulley/belt assembly, a stepper motor and a CLYC detector. One of the advantages of our prototype is interchangeability of collimator masks and main detectors. Masks were fabricated based on shielding calculations performed for various mask configurations, and the timing pulley system was carefully designed to effectively and reliably transfer the work load to the driver tube. Rotation control system was assembled. The rotation of the collimators is controlled by an additionally designed controller switch and the GUI-based software system. The RMC system was tested for its mechanical performance by checking alignments and rotational characteristics, and modulation patterns were obtained with a ${ }^{133}$ Ba gamma-ray source to investigate the repeatability and reproducibility of the pattern acquisition. The prototype RMC system showed rotational accuracy of less than $0.1 \%$ in repeated tests and produced similar modulation patterns from repeated measurements, which can be a promising feature as a radiation imager. The system is ready to be investigated for further 
studies and applications as a dual-particle imager for nuclear security and safeguards.

\section{Conflict of Interest}

The authors declare there is no conflict of interest associated with this article.

\section{Acknowledgements}

This work was supported by the Nuclear Safety Research Program through the Korea Foundation of Nuclear Safety (KOFONS), granted financial resource from the Nuclear Safety and Security Commission (NSSC), Republic of Korea (No. 1403024).

\section{Author Contribution}

Conceptualization: Kim G. Methodology: Kim HS, Bang YB, Ye SJ. Formal analysis: Kim HS, Lee JY, Choi SH. Funding acquisition: Kim G. Project administration: Kim G. Visualization: Bang. Writing - original draft: Kim HS. Writing - review and editing: Kim G. Approval of final manuscript: all authors.

\section{References}

1. Gmar M, Agelou M, Carrel F, Schoepff V. GAMPIX: a new generation of gamma camera. Nucl Instrum Methods Phys Res A. 2011; 652(1):638-640.

2. Wahl CG, Kaye WR, Wang W, Zhang F, Jaworski JM, King A, et al. The Polaris-H imaging spectrometer. Nucl Instrum Methods Phys Res A. 2015;784:377-381.

3. Oda M. High-resolution x-ray collimator with broad field of view for astronomical use. Appl Opt. 1965:4(1):143.

4. Kowash BR, Wehe DK, Fessler JA. A rotating modulation imager for locating mid-range point sources. Nucl Instrum Methods Phys Res A. 2009;602(2):477-483.

5. Sharma AC, Tourassi GD, Kapadia AJ, Harrawood BP, Bender JE, Crowell AS, et al. Design and development of a high-energy gamma camera for use with NSECT imaging: feasibility for breast imaging. IEEE Trans Nucl Sci. 2007;54(5):1498-1505.

6. Kim HS, Ye SJ, Shin Y, Lee G, Kim G. Radiation imaging with a rotational modulation collimator (RMC) coupled to a $\mathrm{Cs}_{2} \mathrm{LiYCl}_{6}: \mathrm{Ce}$
(CLYC) detector. J Korean Phys Soc. 2016;69(11):1644-1650.

7. Kim HS, Choi HY, Lee G, Ye SJ, Smith MB, Kim G. A Monte Carlo simulation study for the gamma-ray/neutron dual-particle imager using rotational modulation collimator (RMC). J Radiol Prot. 2018;38(1):299-309.

8. Kim HS, Smith MB, Koslowsky MR, Kwak SW, Ye SJ, Kim G. Characterization of a CLYC detector and validation of the Monte Carlo simulation by measurement experiments. J Radiat Prot Res. 2017;42(1):48-55.

9. Kim C, Yeom JY, Kim G. Digital n- $\gamma$ pulse shape discrimination in organic scintillators with a high-speed digitizer. J Radiat Prot Res. 2019;44(2):53-63.

10. Goorley T, James M, Booth T, Brown F, Bull J, Cox LJ, et al. Initial MCNP6 release overview. Nucl Technol. 2012;180(3):298-315.

11. Kim HS, Ye SJ, Lee G, Kim G. Optimization of the collimator mask for the rotational modulation collimator-based gamma-ray/neutron dual-particle imager. Curr Appl Phys. 2019;19(7):856-865.

12. Barros S, Kim G. Monte Carlo studies and response assessment of a new Rotational Modulation Collimator (RMC) design. J Radioanal Nucl Chem. 2018;316(3):1119-1128.

13. Barros S, Kim G. Design modifications to Rotating Modulation Collimators for extended field-of-view. Appl Radiat Isot. 2021; 170:109597.

14. Jeong M, Van B, Wells BT, D'Aries LJ, Hammig MD. Scalable gamma-ray camera for wide-area search based on silicon photomultipliers array. Rev Sci Instrum. 2018;89(3):033106.

15. Jeong M, Hammig MD. Comparison of gamma ray localization using system matrixes obtained by either MCNP simulations or ray-driven calculations for a coded-aperture imaging system. Nucl Instrum Methods Phys Res A. 2020;954:161353.

16. Omata A, Kataoka J, Fujieda K, Sato S, Kuriyama E, Kato H, et al. Performance demonstration of a hybrid Compton camera with an active pinhole for wide-band X-ray and gamma-ray imaging. Sci Rep. 2020;10(1):14064.

17. Lehner CE, He Z, Zhang F. $4 \pi$ Compton imaging using a 3-D position-sensitive CdZnTe detector via weighted list-mode maximum likelihood. IEEE Trans Nucl Sci. 2004;51(4):1618-1624.

18. Park S, Kim G, Lee G. Image reconstruction for rotational modulation collimator (RMC) using non local means (NLM) denoising filter. Nucl Instrum Methods Phys Res A. 2020;954:161901.

19. Shin Y, Kim G, Lee G. Improving image reconstruction algorithm for rotating modulation collimators using a variance stabilizing transform. J Instrum. 2017;12:C01051.

20. Shin Y, Kim G, Lee G. Regularizing RMC images for locating midrange point sources. J Instrum. 2016;11:C12007. 